Department of Ophthalmology, Faculty of Medicine Siriraj Hospital, Mahidol University, Bangkok, Thailand P Prabhasawat

Department of Ophthalmology, Pramongkutklao Hospital, Bangkok, Thailand

$\mathrm{N}$ Tesavibul

Department of Ophthalmology, Metapracharak

Hospital, Bangkok, Thailand

W Komolsuradej

Correspondence to: Pinnita Prabhasawat, MD, Department of Ophthalmology, Siriraj

Hospital Mahidol University, Bangkok, Thailand

sippb@mahidol.ac.th

Accepted for publication 8 August 2001

\title{
Single and multilayer amniotic membrane transplantation for persistent corneal epithelial defect with and without stromal thinning and perforation
}

\author{
Pinnita Prabhasawat, Nattaporn Tesavibul, Wiwat Komolsuradej
}

\begin{abstract}
Aims-To evaluate the efficacy of amniotic membrane transplantation (AMT) in persistent corneal epithelial defect with or without stromal thinning and corneal perforation.

Methods-28 patients (28 eyes) with persistent corneal epithelial defect unresponsive to medical treatment were given preserved human amniotic membrane transplants. The patients were divided into three groups: group A, persistent corneal epithelial defect 10 eyes; group $B$, epithelial defect with stromal thinning 13 eyes; and group $\mathrm{C}$, corneal perforation five eyes. AMT was performed using one layer in group $A$ and multilayers in group $B$ and $C$. The causes of persistent epithelial defect were neurotrophic keratopathy (24 eyes), limbal deficiency (six eyes), exposure keratopathy (four eyes), and Mooren's ulcer (one eye).

Results-Success was noted in $82.1 \%$ (23/28 eyes) in all groups, with $80 \%(8 / 10$ eyes), $84.6 \%$ ( $11 / 13$ eyes), and $80 \%$ (4/5 eyes) in groups $\mathrm{A}, \mathrm{B}$, and $\mathrm{C}$ respectively, with a mean follow up of 10.9 months (1-30 months). The mean epithelialisation time after AMT was 2.1 weeks. The healing times of groups $B$ and $C$ are also significantly shorter than group $A(p=0.017$ and 0.018 , respectively). Corneal stromal thickness was significantly increased in all cases in groups $B$ and $C(p=0.006)$. Those with corneal perforation in group $C$ were completely healed by multilayer AMT. There was no difference in the epithelialisation time between successful cases treated by a single operation (17 eyes) or repeated operation (six eyes). Vision improved in $18.9 \%(8 / 28$ eyes) and worsened as a result of cataract formation in $2.3 \%$ (1/28 eyes). Failure was noted in $17.9 \%$ (5/28 eyes), because of corneal infection (two eyes), neurotrophic keratopathy with and without limbal deficiency (two eyes), and intractable corneal perforation (one eye). No patient developed major immediate postoperative complications or graft rejection.
\end{abstract}

Conclusion-Amniotic membrane can successfully treat refractory corneal epithelial defect by promoting epithelial healing and thus prevent corneal perforation. It can be used as a treatment for corneal perforation by restoring corneal stromal thickness so that emergency penetrating keratoplasty can be avoided. (Br f Ophthalmol 2001;85:1455-1463)

An intact corneal epithelium is one of the most important factors in maintaining ocular surface health. Corneal epithelial defects usually heal without any complications by a rapid proliferation of epithelial cells. During the process, several factors, such as chemical injuries from topical medications, mechanical insults from lid abnormalities, xerosis and trauma, and a deprivation of sensory innervation - that is, a neurotrophic keratopathy, can interfere with wound healing, leading to a condition called persistent epithelial defect (PED). ${ }^{1}$ Once PED occurs and is left unattended, Dellen's ulcer, corneal melting, descemetocele, and corneal perforation can subsequently develop, leading to a devastating condition and loss of sight. ${ }^{2}$ Traditional treatment for PED includes correcting the underlying condition, suppressing the inflammation, and promoting the healing process. Current treatments include tears, lubricants, fibronectin, ${ }^{34}$ growth factors, ${ }^{5}$ and substance P. ${ }^{6}$ However, if the defect persists even after the application of these treatments and stromal ulcer and thinning develop, surgical treatment such as tissue adhesive, ${ }^{7}$ contact lens, ${ }^{8}$ conjunctival flap, ${ }^{9}$ and tarsorrhaphy ${ }^{10}$ are often tried. Once a corneal perforation occurs application of glue and/or a penetrating keratoplasty is usually performed to restore the globe integrity. Nevertheless, these treatments are not without problems - for example, decreased vision and poor cosmesis with a conjunctival flap and tarsorrhaphy, tissue inflammation from glue toxicity, and an inadequacy of donated corneas in some countries.

In 1997, Lee and Tseng first introduced the use of amniotic membrane in the treatment of PED with corneal ulcer. ${ }^{11}$ The membrane has been used successfully to treat PED and ulcers 

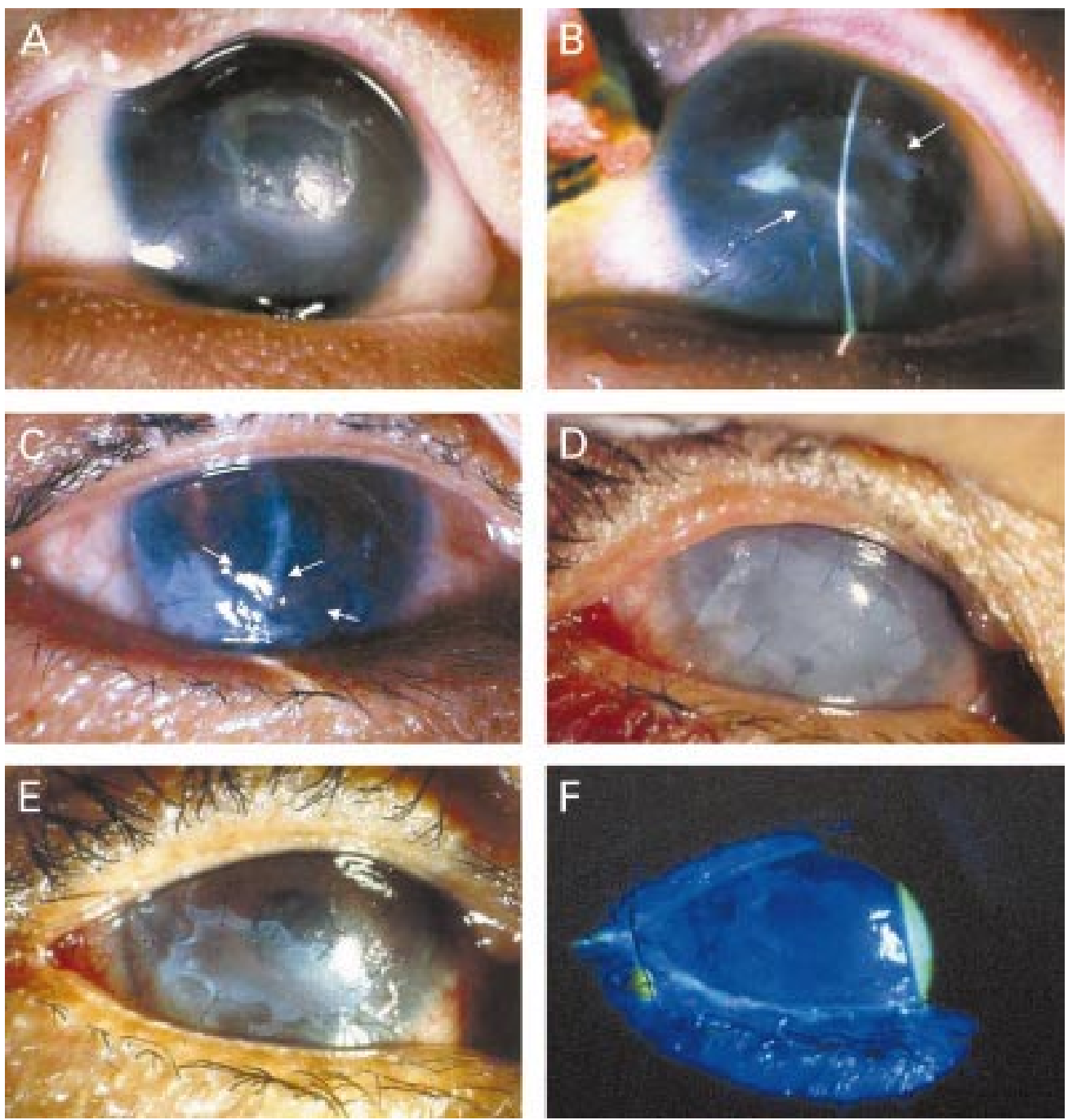

Figure 1 Group A. A patient with rhabdomyosarcoma $(A)$ of the eyelid who underwent tumour removal surgery and radiation resulting in upper eyelid defect, with exposure keratopathy and a persistent epithelial defect. Two weeks after AMT and nasal tarsorrhaphy, part of the membrane started to dissolve and the epithelium healed completely (B). A patient with a history of recurrent herpes simplex keratitis $(C)$ and post phacoemulsification, developed a persistent corneal epithelial defect over 1 year. After single layer AMT, the epithelial defect healed completely in 8 weeks but recurred after the amniotic membrane partially dissolved (arrows). A single layer AMT combined with an amniotic membrane patch and tarsorrhaphy were performed (D). One week after AMT, part of the patch dissolved (E). Complete epithelial healing as shown by fluorescein staining $(F)$, which remained stable 5 months thereafter, until optical PKP was performed.

from different causes. The procedure has been shown to promote epithelial healing, reduce vascularisation, yield a good cosmesis, and also to be relatively easy to perform. Kruse et $a l^{12}$ also reported a rapid healing of the epithelial defect with an increase in stromal thickness after multilayer amniotic membrane transplantation (AMT) for deep corneal ulcers. Recently, Tseng and colleagues reported their success in using amniotic membrane (single and multilayer as a graft and patch) in persistent and progressive neurotrophic corneal ulcers. It has not been reported, but would be interesting to know the difference in corneal healing using a single layer of membrane and a multilayer method in PED and PED with stromal thinning and/or perforation. To answer these questions, we summarise our experience in using AMT for PED with and without corneal thinning, descemetocele, and perforation.

\section{Patients and methods}

PATIENTS

This study was approved by the committee for the protection of human subjects in research, a part of the faculty of medicine at Siriraj Hospital, in Bangkok, Thailand. Between September 1997 and December 2000, 42 AMTs were performed on 28 patients (28 eyes). Written informed consent was obtained from each patient. Criteria for recruitment were persistent corneal epithelial defect that failed to respond to conventional treatments, PED with descemetocele or stromal thinning, and PED with a corneal perforation smaller than $1 \mathrm{~mm}$ in diameter with positive Seidel test. All patients were referred by different ophthalmologists after conventional treatments had been tried without success. The patients were divided into three groups-group A, PED without stromal thinning (10 eyes in 10 patients; see Fig 1); group B, PED with stromal thinning or descemetocele (13 eyes in 

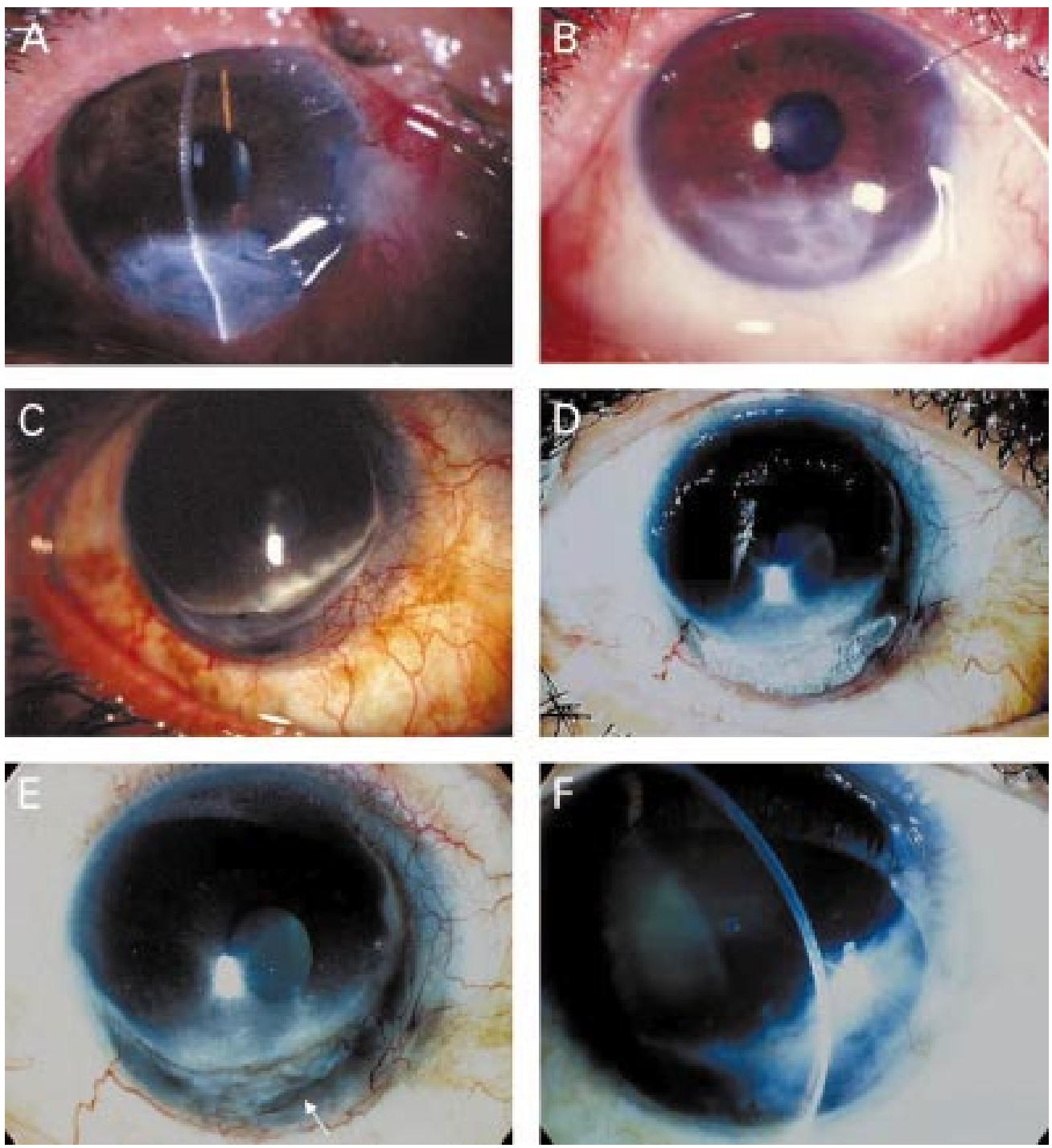

Figure 2 Group B. A patient developed a descemetocele from exposure keratopathy after wide excision of a squamous cell carcinoma of the lower eyelid. Appearance at 7 days after multilayer AMT revealed no epithelial defect $(A)$. One month after surgery, the eye showed an increased stromal thickness and a marked reduction of ocular inflammation (B). Surface integrity was maintained up to 9 months before advanced recurrent tumours developed which required exenteration. A patient with Mooren's ulcer developed a corneal perforation (C). Immunosuppressive treatment and glue were used to patient with Mooren's ulcer developed a corneal perforation (C). Immunosuppressive treatment and glue were used to
restore the globe integrity and reduce inflammation (D). Because of the irritative symptoms and glue toxicity, which induced chronic inflammation and prevented tissue healing, the glue was removed and a descemetocele developed which required a multilayer AMT. Complete epithelialisation after 7 postoperative days with a partially dissolved membrane (arrow) (E). Four months after surgery, the eye showed an increased stromal thickness with no inflammation $(F)$.

13 patients; see Fig 2); and group C, PED with corneal perforations (five eyes in five patients; see Fig 3). Surgical success was defined as complete epithelialisation of the ocular surface at the operated site as demonstrated by biomicroscopy and negative fluorescein staining without recurrence at the same area within 1 month after healing. In cases with corneal perforation (group C) surgical success also includes cessation of aqueous leakage and formation of a deep anterior chamber for at least 1 month postoperatively. Failure was defined as incomplete epithelialisation, persistence of the defect, or recurrence of an epithelial defect at the same site. In group $\mathrm{C}$ failure also included recurrence of aqueous leakage. Corneal thickness was evaluated using biomicroscopy before and after the operation at each visit. Stromal thickness at the thinning area (group B) or perforated site (group C) was recorded and compared with the thickness of the same area after the operation only when the surgical area was completely epithelialised. Increase in stromal thickness of the eye at the last follow up as seen by biomicroscopy and photography is defined as improvement of stromal thickness.

All patients had one or more underlying disease which predisposed to epithelial break down and PED-for example, neurotrophic keratopathy, limbal stem cell deficiency, exposure keratopathy, and Mooren's ulcer. Several associated conditions perpetuated the corneal epithelial defects such as severe keratoconjunctivitis sicca, bullous keratopathy, and keratouveitis. The demographic and clinical data are summarised in Tables 1 and 2 . 

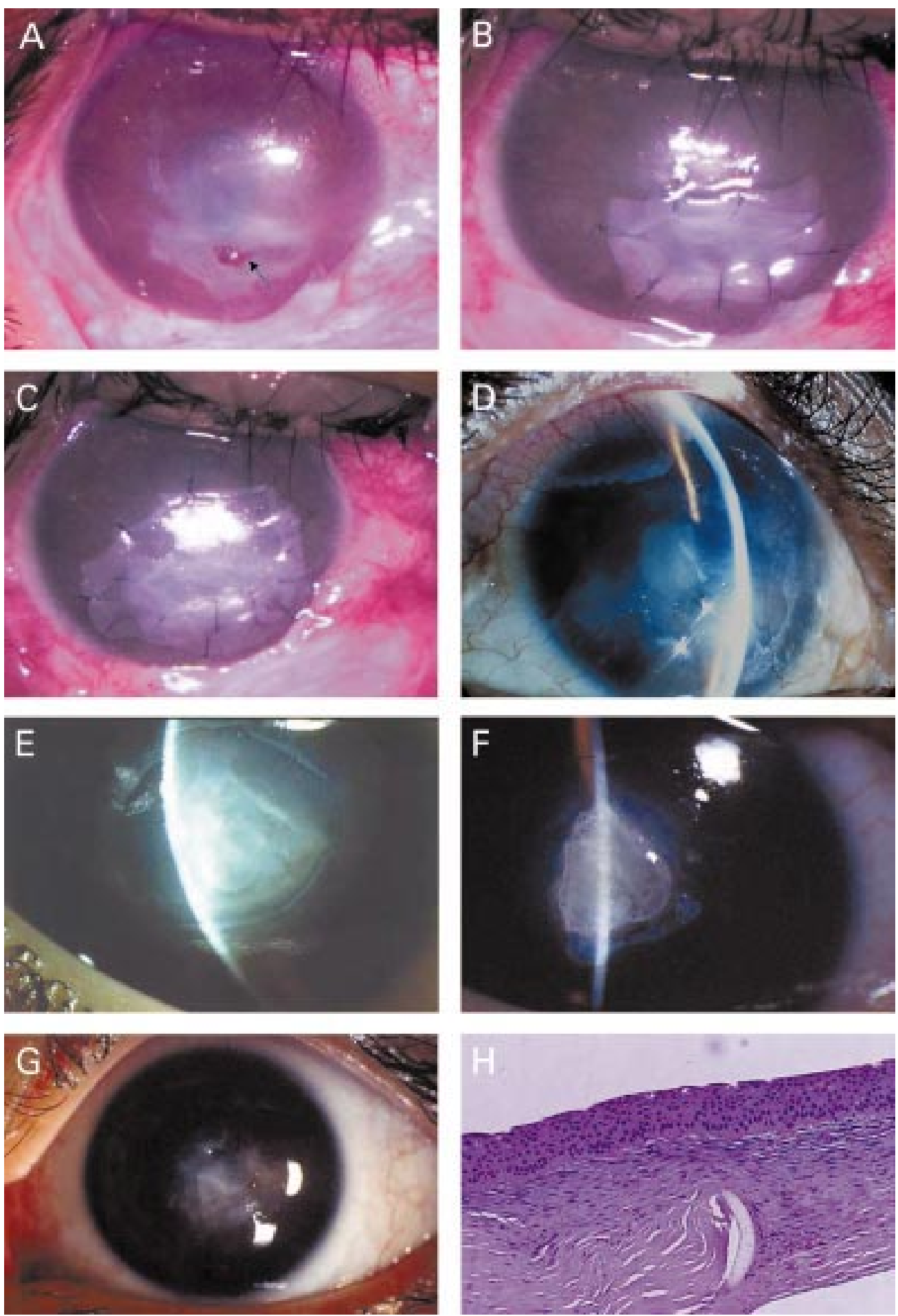

Figure 3 Group C. A patient with a history of recurrent herpes simplex virus keratitis developed a corneal perforation $(A$, arrow). A multilayer AMT was performed (B) with an amniotic patch on top (C). Four months postoperatively, a membrane completely dissolved revealing a fully restored stromal thickness with no epithelial defect (D). The corneal pannus resulted from a previous inflammation induced by herpes. A $3 \mathrm{~mm}$ corneal perforation in an eye with herpetic keratitis superimposed by bacterial corneal ulcer was managed by glue and multilayer AMT (E). The membrane dissolved totally after 1 month $(F)$. The exposed glue induced corneal vascularisation and interfered with corneal healing. Removal of glue and repeated multilayer AMT decreased the corneal vascularisation and increased stromal thickness to normal (G). Five months later, PKP was performed which improved the vision from counting fingers to 20/50. A pathological section revealed a normal stromal thickness with abundant keratocytes and a residual part of the membrane in the area of perforation $(H)$.

PREPARATION OF PRESERVED HUMAN AMNIOTIC MEMBRANE

Amniotic membranes obtained from the Bangkok Biomaterial Center were used for all patients in this study. Human amniotic membrane was prepared and preserved using a method previously described by Lee and Tseng ${ }^{11}$ with minor modification. ${ }^{14}$ The sheets of membranes were stored at $-70^{\circ} \mathrm{C}$ for up to 1 year before use. 
Table 1 Demographic data

\begin{tabular}{|c|c|c|c|c|}
\hline & Group $A$ & Group B & Group $C$ & Total \\
\hline No (eyes) & 10 & 13 & 5 & 28 \\
\hline Age (year) & $50.2(25.6)(5-80)$ & $56.2(17.5)(30-86)$ & $25.2(14.1)(7-38)$ & $48.7(22.7)(5-86)$ \\
\hline $\operatorname{Sex}(M / F)$ & $7 / 3$ & $5 / 8$ & $4 / 1$ & $16 / 12$ \\
\hline \multicolumn{5}{|l|}{ Cause of PED ${ }^{\star}$} \\
\hline Neurotrophic/LD/exposure K/Mooren's ulcer & $9 / 3 / 1 /-$ & $11 / 1 / 3 / 1$ & $4 / 2 /-1-$ & $24 / 6 / 4 / 1$ \\
\hline \multicolumn{5}{|l|}{ Aggravating conditions ${ }^{\star}$} \\
\hline Bullous keratitis and graft rejection & 5 & 4 & 1 & 10 \\
\hline KCS & 4 & 6 & 2 & 12 \\
\hline Radiation & 1 & 3 & - & 4 \\
\hline $\mathrm{DM}$ & 3 & 2 & - & 5 \\
\hline SJS & 1 & 1 & 2 & 4 \\
\hline HSK & 1 & 2 & 2 & 5 \\
\hline Bacterial/fungal infection & $1 / 1$ & $2 /-$ & $2 /-$ & $5 / 1$ \\
\hline Chemical burn/trauma & $1 /-$ & -13 & $-1-$ & $1 / 3$ \\
\hline Keratouveitis & - & 1 & - & 1 \\
\hline \multicolumn{5}{|l|}{ Previous surgeries } \\
\hline Phacoemulsification & 2 & 3 & - & 5 \\
\hline $\mathrm{PKP} / \mathrm{PKP}+\mathrm{LT}$ & 2 & - & 1 & 3 \\
\hline Lid surgery for tumour removal & 1 & 2 & - & 3 \\
\hline Glaucoma surgery & 1 & 1 & - & 2 \\
\hline Pterygium excision/band keratopathy removal/glue & $-1-1-$ & $1 / 1 / 1$ & $-/-1-$ & $1 / 1 / 1$ \\
\hline \multicolumn{5}{|l|}{ Previous management } \\
\hline Soft contact lens & 4 & 5 & - & 9 \\
\hline Patching & 4 & 2 & - & 6 \\
\hline Tear supplement & 8 & 9 & 1 & 18 \\
\hline Punctal occlusion & 2 & 3 & 2 & 7 \\
\hline Tarsorrhaphy & 2 & - & 1 & 3 \\
\hline Follow up & $12.8(9.3)(3-30)$ & $10.9(4.1)(5-15)$ & $7.5(7.6)(1-20)$ & $10.9(6.9)(1-30)$ \\
\hline
\end{tabular}

* Some cases had more than one condition. PED = persistent epithelial defect, Exposure $\mathrm{K}=$ exposure keratopathy, Bullous $\mathrm{K}=$ bullous keratopathy, $\mathrm{KCS}=$ keratoconjunctivitis sicca, $\mathrm{DM}=$ diabetic mellitus, $\mathrm{SJS}=$ Stevens-Johnson syndrome, HSK $=$ herpes simplex keratitis, $\mathrm{PKP}=$ penetrating keratoplasty, $\mathrm{LT}=$ limbal transplantation, $\mathrm{LD}=$ limbal stem cell deficiency.

AMNIOTIC MEMBRANE TRANSPLANTATION, SURGICAL TECHNIQUE

All surgery was performed by one surgeon (PP). After retrobulbar or topical anaesthesia, amniotic membranes were placed using different techniques to cover the areas of defect in the recipient eyes. In group A (PED without stromal thinning) a single layer of membrane was used to cover the defect by trimming off the excess parts and was secured to the edge of the defect by interrupted or continuous 10-0 nylon suture with buried knots. The membrane was placed stromal side down (fig 1D). For group B (PED with stromal thinning) amniotic membranes were placed stromal side down, layer by layer, to fill up the ulcer and cover the defect. The layers were trimmed to fit the ulcer and the top layer was sutured as described above; the bottom layers were left unsutured (Fig 2A). Two patients in group B underwent a single layer technique as in group A because of minimal stromal thinning. For group $C$, the bottom of a perforated site was covered by a small piece of amniotic membrane (AM) that fitted the size of the perforation and multilayer $\mathrm{AM}$ was then applied layer by layer to fill up the thinning area (Fig 3B). A larger piece of $\mathrm{AM}$ was placed uppermost to cover the whole defect area, trimmed to fit the ulcer, and sutured with an interrupted 10-0 nylon with an attempt to include a few layers of AM (Fig 3C). In some severe neurotrophic keratopathy cases, we performed a patch technique by applying an amniotic membrane over the whole cornea with the basement membrane side facing down, and sutured with $10-0$ nylon as described. A bandage contact lens was applied on top of the membrane until the epithelial defect was completely healed.

POSTOPERATIVE EVALUATION AND FOLLOW UP All patients received either topical $1 \%$ prednisolone acetate (Predforte, Allergan, Irvine,
CA, USA) or non-preservative $1 \%$ methylprednisolone and $0.3 \%$ ciprofloxacin (Ciloxan, Alcon, Forte Worth, TX, USA) four times daily until complete epithelialisation was noted. After healing, topical antibiotics were discontinued and the topical steroid was tapered off.

Assessment of surgical outcome was determined by patients' symptoms (particularly pain, discomfort, and irritation), inflammation of the cornea and amniotic graft, healing time of the area covered by the membrane, corneal thickness, and cosmetic appearance. Fluorescein staining was used to detect epithelial defects. All patients were examined on postoperative day 1 , then at the end of the first week, second week, and monthly thereafter. Corneal epithelial healing was further classified into success and failure.

\section{STATISTICAL ANALYSIS}

The qualitative data were analysed by $\chi^{2}$ test. The quantitative preoperative and postoperative data were analysed by paired sample $t$ tests for the parametric data and Wilcoxon sign rank test for non-parametric data. In independent nonparametric data, a Mann-Whitney U test was used to compare the two groups and KruskalWallis $\mathrm{H}$ was used to compare more than two groups and ANOVA in parametric data. The data were analysed using SPSS version 9.0 with the assistance of the statistics unit of the department of ophthalmology, Siriraj Hospital. A p value of less than 0.05 was considered significant.

\section{Results}

The mean age of these 28 patients ( 16 men and 12 women) was 48.7 years (range 5-86 years). Total mean follow up time was 10.9 (SD 6.9) months (range 1-30 months), and 12.8 (9.3) months (3-30 months), 10.9 (4.1) months (5-15 months), and 7.5 (7.6) months (1-20 months), in groups $\mathrm{A}, \mathrm{B}$, and $\mathrm{C}$ respectively (Table 1$)$. The 
Table 2 Details of clinical data

\begin{tabular}{|c|c|c|c|c|c|c|c|c|c|c|c|}
\hline \multicolumn{2}{|c|}{ Case Age } & Sex & Main diagnosis & Associated disease & $\begin{array}{l}\text { Previous Rx of } \\
E D\end{array}$ & $\begin{array}{l}\text { ED Size } \\
(\mathrm{mm})\end{array}$ & $\begin{array}{l}\text { Epithelialisation } \\
\text { (weeks) }\end{array}$ & Surgery & $\begin{array}{l}F / U \\
(M)\end{array}$ & Result & Remarks \\
\hline \multicolumn{12}{|c|}{ Group A } \\
\hline 1 & 80 & $\mathrm{~F}$ & Neurotrophic & PBK & SCL, tear & 3 & 3 & $\mathrm{AM}$ & 24 & $\mathrm{~S}$ & \\
\hline 2 & 48 & M & Neurotrophic & Graft failure post PKP & SCL, tear & 3 & 1 & $\mathrm{AM}$ & 6 & $\mathrm{~S}$ & \\
\hline 3 & 69 & $\mathrm{~F}$ & Neurotrophic & PBK, HSK, KCS, DM & SCL, tear, $\mathrm{PO}$ & 6 & 8 & $\mathrm{AM}$ & 7 & $\mathrm{~S}$ & PKP at 7 th month \\
\hline \multicolumn{6}{|c|}{2 nd AM, new site at 4 th month } & 4 & 1 & $\begin{array}{l}\text { AM and patch } \\
\text { tarsorrhaphy }\end{array}$ & & $\mathrm{S}$ & \\
\hline 4 & 5 & M & Neurotrophic, LD & SJS & Tear, patch & 3 & 6 & $\mathrm{AM}$ & 30 & $\mathrm{~S}$ & LT at 24 th month \\
\hline 5 & 70 & $M$ & Neurotrophic & $\mathrm{BK}, \mathrm{KCS}, \mathrm{DM}$ & Tear, PO & 2 & 8 & $\mathrm{AM}$ & 19 & $\mathrm{~S}$ & \\
\hline 6 & 58 & $\mathrm{~F}$ & Neurotrophic & $\mathrm{BK}$, post bacterial ulcer & Patch & 2 & 1 & $\mathrm{AM}$ & 6 & $\mathrm{~S}$ & \\
\hline 7 & 9 & M & Exposure & $\begin{array}{l}\text { Lid abnormality after } \\
\text { tumour removal, post } \\
\text { radiation, KCS }\end{array}$ & $\begin{array}{l}\text { Tear, patch, } \\
\text { tarsorrhaphy }\end{array}$ & 4 & 2 & AMs tarsorrhaphy & 18 & $\mathrm{~S}$ & $\begin{array}{l}\text { Tarsorrhaphy separation } \\
\text { at } 20 \text { th week }\end{array}$ \\
\hline \multicolumn{6}{|c|}{ 2nd AM, new site at 6 th month } & 3 & 3 & AMs tarsorrhaphy & & $S$ & \\
\hline \multicolumn{6}{|c|}{ 3rd AM, new site at 7 th month } & 3 & 3 & $\mathrm{AM}$ & & $\mathrm{s}$ & \\
\hline 8 & 68 & $\mathrm{M}$ & Neurotrophic, LD & $\begin{array}{l}\text { PKP, trabeculectomy } 5 \\
\text { times, KCS, DM }\end{array}$ & SCL, tear & 7 & 4 (not healed) & $\mathrm{AM}$ and patch & 3 & $\mathrm{~F}$ & $\begin{array}{l}\text { LT }+ \text { PKP at } 3 \text { rd month, } \\
\text { ED healed in } 8 \text { days }\end{array}$ \\
\hline \multicolumn{6}{|c|}{ 2nd AM, same site at 1 st month } & 3 & 8 (not healed) & AMs tarsorrhaphy & & $\mathrm{F}$ & \\
\hline 9 & 40 & M & Neurotrophic, LD & $\begin{array}{l}\text { Acute burn, lid } \\
\text { abnormality }\end{array}$ & $\begin{array}{l}\text { Tear, } \\
\text { tarsorrhaphy }\end{array}$ & 5 & 3 & AMs tarsorrhaphy & 5 & $\mathrm{~S}$ & \\
\hline \multicolumn{6}{|c|}{2 nd AM, same site at 0.5 th month } & $\begin{array}{l}5 \\
5\end{array}$ & $\begin{array}{l}2 \text { (not healed) } \\
2 \text { (not healed) }\end{array}$ & $\begin{array}{l}\mathrm{AM} \\
\mathrm{AM}\end{array}$ & 10 & $\begin{array}{l}\mathrm{F} \\
\mathrm{F}\end{array}$ & Healed with antifungus \\
\hline \multicolumn{12}{|c|}{ Group B } \\
\hline 1 & 33 & M & Neurotrophic & $\begin{array}{l}\text { Post bacterial ulcer, post } \\
\text { trauma }\end{array}$ & Tear & 4 & 1 & AM 2 layers & 14 & $\mathrm{~S}$ & \\
\hline 2 & 85 & $\mathrm{~F}$ & Neurotrophic & HSK, KCS, DM & PO, SCL, tear & 3 & 3 & $\mathrm{AM}$ & 15 & $\mathrm{~S}$ & \\
\hline 3 & 58 & $M$ & Neurotrophic & Post bacterial ulcer, BK & Tear & 3 & 4 & AM 2 layers & 5 & $\mathrm{~S}$ & \\
\hline 4 & 64 & $\mathrm{~F}$ & $\begin{array}{l}\text { Neurotrophic } \\
\text { (descemetocele) }\end{array}$ & $\begin{array}{l}\text { KCS, post radiation, } \\
\text { uveitis, cataract }\end{array}$ & PO, tear & 3 & 1 & AM 2 layers & 6 & $S$ & $\begin{array}{l}\text { Enucleation due to } \\
\text { painful eye at } 6 \text { th month }\end{array}$ \\
\hline \multicolumn{6}{|c|}{ 2nd AM, new site at 0.5 th month } & 3 & 1 & AM 2 layers & & $\mathrm{S}$ & \\
\hline \multicolumn{6}{|c|}{ 3rd AM, new site at 1st month } & 2 & 1 & AM 3 layers & & $\mathrm{S}$ & \\
\hline \multicolumn{6}{|c|}{4 th $\mathrm{AM}$, new site at 1 st month } & 2 & 1 & AM 2 layers & & $\mathrm{s}$ & \\
\hline \multicolumn{6}{|c|}{5 th op, new site at 1 st month } & 2 & 1 & AM 2 layers & & $\mathrm{S}$ & \\
\hline 5 & 61 & $\mathrm{~F}$ & $\begin{array}{l}\text { Neurotrophic } \\
\text { (descemetocele) }\end{array}$ & $\begin{array}{l}\text { Post trauma, post } \\
\text { phacoemulsification }\end{array}$ & Tear, patch & 3 & 3 & AM 2 layers & 13 & $\mathrm{~S}$ & \\
\hline 6 & 50 & $\mathrm{~F}$ & Neurotrophic & Post trauma, BK, DM & Tear & 3 & - & AM 3 layers & 13 & $\mathrm{~F}$ & Lost AMT in 1 week \\
\hline \multicolumn{6}{|c|}{ 2nd AM, same site at 1 st month } & 3 & 1 & AM 2 layers & & $S$ & \\
\hline 7 & 86 & $\mathrm{~F}$ & Neurotrophic & $\begin{array}{l}\text { KCS, HSK, post remove } \\
\text { band keratopathy }\end{array}$ & SCL, patch & 3 & 3 (not healed) & $\begin{array}{l}\text { AM } 2 \text { layers and } \\
\text { patch }\end{array}$ & 15 & $\mathrm{~F}$ & $\begin{array}{l}\text { Healed with topical } \\
\text { aciclovir in } 1 \text { week }\end{array}$ \\
\hline 8 & 47 & $\mathrm{~F}$ & $\begin{array}{l}\text { Neurotrophic, } \\
\text { exposure K } \\
\text { (descemetocele) }\end{array}$ & $\begin{array}{l}\text { Post radiation, lid } \\
\text { abnormal post tumor } \\
\text { removal }\end{array}$ & & 4 & 1 & AM 2 layers & 15 & $\mathrm{~S}$ & $\begin{array}{l}\text { Exenteration at } 15 \text { th } \\
\text { month }\end{array}$ \\
\hline 9 & 41 & M & $\begin{array}{l}\text { Neurotrophic, } \\
\text { exposure K } \\
\text { (descemetocele) }\end{array}$ & $\begin{array}{l}\text { Lid abnormal post } \\
\text { radiation, CA } \\
\text { nasopharynx, KCS, S/P } \\
\text { trabeculectomy }\end{array}$ & Tear & 3 & 1 & $\begin{array}{l}\text { AM } 2 \text { layers + } \\
\text { tarsorrhaphy }\end{array}$ & 10 & $\mathrm{~S}$ & \\
\hline \multicolumn{5}{|c|}{ 2nd AM, new site at 1.5 th month } & - & 3 & & AM 3 layers & & $\mathrm{S}$ & \\
\hline 10 & 56 & $\mathrm{M}$ & $\begin{array}{l}\text { LD } \\
\text { (descemetocele) }\end{array}$ & SJS, KCS & Tear, PO & 4 & 1 & $\begin{array}{l}\text { AM } 2 \text { layers and } \\
\text { patch }\end{array}$ & 5 & $S$ & \\
\hline 11 & 52 & $\mathrm{~F}$ & $\begin{array}{l}\text { Neurotrophic } \\
\text { (descemetocele) }\end{array}$ & PBK & CL & 4 & 2 & $\mathrm{AM}$ & 9 & $\mathrm{~S}$ & \\
\hline 12 & 30 & $\mathrm{~F}$ & $\begin{array}{l}\text { Mooren's ulcer } \\
\text { (descemetocele) }\end{array}$ & & Glue, CL & 10 & 1 & $\begin{array}{l}\text { AM } 3 \text { layers + } \\
\text { remove glue }\end{array}$ & 6 & $\mathrm{~S}$ & \\
\hline 13 & 73 & $\mathrm{~F}$ & $\begin{array}{l}\text { Neurotrophic, } \\
\text { exposure } \\
\text { (descemetocele) }\end{array}$ & $\begin{array}{l}\mathrm{KCS}, \mathrm{ABK} \text {, post } \\
\text { cataract extraction and } \\
\text { pterygium excision }\end{array}$ & CL, tear & 4 & 2 (not healed) & AM 3 layers & 11 & $\mathrm{~F}$ & AM loss at 2 nd week \\
\hline \multicolumn{6}{|c|}{ 2nd AM, same site at 0.5 th month } & 4 & 4 (not healed) & AM 3 layers & & $\mathrm{F}$ & $\begin{array}{l}\text { Healed with patch in } 1 \\
\text { week }\end{array}$ \\
\hline \multicolumn{12}{|c|}{ Group C } \\
\hline 1 & 38 & $M$ & Neurotrophic, LD & $\begin{array}{l}\text { SJS, KCS, S/P LT, PKP } \\
\text { graft rejection }\end{array}$ & $\begin{array}{l}\text { PO, tear, } \\
\text { tarsorrhaphy }\end{array}$ & 3 & 0.5 & AM 3 layers & 1 & $\mathrm{~S}$ & $\begin{array}{l}\text { PKP at 1st month for } \\
\text { vision }\end{array}$ \\
\hline 2 & 34 & $M$ & $\mathrm{LD}$ & SJS, KCS & $\mathrm{PO}$ & 3 & 1 & AM 3 layes & 20 & $S$ & \\
\hline 3 & 7 & $\mathrm{~F}$ & Neurotrophic & $\begin{array}{l}\text { HSK, post bacterial } \\
\text { ulcer }\end{array}$ & - & 6 & 2 & AM 3 layers + glue & 5 & $\mathrm{~S}$ & $\begin{array}{l}\text { Remove glue at } 1.5 \text { th } \\
\text { month }\end{array}$ \\
\hline \multicolumn{6}{|c|}{ 2nd op, 1.5 th month (after glue removal) } & 6 & 1 & AM 3 layers & & $\mathrm{S}$ & $\begin{array}{l}\mathrm{PKP} \text { at } 5 \text { th month for } \\
\text { vision }\end{array}$ \\
\hline 4 & 13 & M & Neurotrophic & $\begin{array}{l}\text { Post bacterial ulcer, } \\
\text { leukoma }\end{array}$ & - & 3 & 1 & AM 3 layers & 2.5 & $\mathrm{~F}$ & $\begin{array}{l}\text { Aqueous leak twice, AM } \\
\text { slip, PKP at } 2.5 \text { th month }\end{array}$ \\
\hline \multicolumn{6}{|c|}{ 2nd AM, same site at 1 st month } & 3 & 1 & AM 3 layers & & $\mathrm{F}$ & \\
\hline 5 & 34 & $M$ & Neurotrophic & HSK & - & 6 & 1 & AM 6 layers & 11 & $\mathrm{~S}$ & \\
\hline
\end{tabular}

$\mathrm{ED}=$ epithelial defect, $\mathrm{Rx}=$ treatment, $\mathrm{PBK}=$ pseudophakic bullous keratopathy, $\mathrm{SCL}=$ soft contact lens, $\mathrm{AM}=$ amniotic membrane, $\mathrm{PKP}=$ penetrating keratoplasty, HSK = herpes simplex keratitis, $\mathrm{KCS}=$ keratoconjunctivitis sicca, $\mathrm{DM}=$ diabetic mellitus, $\mathrm{PO}=$ punctal occlusion, $\mathrm{SJS}=\mathrm{Stevens-Johnson}$ syndrome, $\mathrm{BK}=$ bullous keratopathy, $\mathrm{LD}=$ limbal stem cell deficiency, $\mathrm{ABK}=$ aphakic bullous keratopathy, $\mathrm{LT}=$ limbal transplantation.

short follow up periods of less than 3 months in three cases (case 8 in group A, cases 1 and 4 in group C) were the result of concurrent procedures, such as penetrating keratoplasty (PKP) and limbal transplantation combined with PKP, which made observation of the corneal surface integrity after AMT no longer possible (Tables 1 and 2). However, the follow up times among these three groups were not statistically significant ( $\mathrm{p}=0.395$ by ANOVA test).
After operation, final vision remained unchanged in 19 eyes $(67.8 \%)$, improved in eight eyes $(28.6 \%)$, and was lost in one eye because of severe uveitis. Among patients with improved vision, five cases $(62.5 \%)$ gained more than 2 Snellen lines. However, because of the severe underlying diseases previously mntioned which led to PED and associated conditions such as glaucoma, cataract, uveitis, and optic neuropathy, the final postoperative 
Table 3 Clinical results

\begin{tabular}{|c|c|c|c|c|c|c|}
\hline & Group $A$ & & Group B & Group $C$ & Total & $p$ Value \\
\hline \multicolumn{7}{|l|}{ Result } \\
\hline Success (\%) & $80(8 / 10)$ & & $84.6(11 / 13)$ & $80(4 / 5)$ & $82.1(23 / 28)$ & 0.55 (Pearson $\chi^{2}$ test) \\
\hline Failure & $20(2 / 10)$ & & $15.4(2 / 13)$ & $20(1 / 5)$ & $17.9(5 / 28)$ & \\
\hline Epithelial defect size (mm) (SD) (range) & $3.9(1.4)(2-7)$ & & $3.5(1.6)(2-10)$ & $4.3(1.6)(3-6)$ & $3.8(1.5)(2-10)$ & 0.44 (Kruskall-Wallis) \\
\hline Epithelialisation time (weeks) (SD) (range) & $3.5(2.6)(1-8)$ & & $1.5(1.0)(1-4)$ & $1.1(0.5)(0.5-3)$ & $2.1(1.9)(0.5-8)$ & $\begin{array}{l}0.003 \text { (total) (Kruskall-Wallis) } \\
0.017 \text { (A and B) (Mann-Whitney } \\
0.009 \text { (Aand C) (Mann-Whitney) }\end{array}$ \\
\hline Stromal thinning (\%) & - & & & & & \\
\hline Preoperative & & & $77.1(36.8)$ & 100 & $84.0(23.9)$ & 0.022 (total) (paired t test) \\
\hline Postoperative & & & $36.7(33.0)$ & $48.3(39.7)$ & $43.7(30.3)$ & 0.018 (group B) \\
\hline Improvement & & & $40.3(26.4)$ & $51.7(39.7)$ & $40.2(28.5)$ & 0.024 (group C) \\
\hline \multicolumn{7}{|l|}{ VA pre/postop } \\
\hline Improve & $2(>2$ line $=1)$ & & $5(>2$ line $=3)$ & $1(>2$ line $=1)$ & $8(>2$ line $=5)$ & \\
\hline Same & 8 & & 7 & 4 & 19 & \\
\hline Worse & - & & 1 & - & 1 & \\
\hline Techniques of AMT & & & & & & Healing in single/multiple layer in \\
\hline Single/multilayer/patch & $14 /-/ 2$ & & $2 / 18 /-$ & $-/ 7 /-$ & $14 / 27 / 4$ & $\begin{array}{l}\text { success cases } \mathrm{p}=0.014 \\
\text { (Mann-Whitney) }\end{array}$ \\
\hline \multicolumn{7}{|l|}{ No of AMT operation } \\
\hline Single/repeated operation & $6 / 4$ & & $9 / 4$ & $3 / 2$ & $18 / 10$ & \\
\hline \multicolumn{7}{|l|}{ Combined procedures } \\
\hline Tarsorrhaphy/glue & $4 /-$ & & $1 /-$ & $-/ 1$ & & \\
\hline \multirow[t]{2}{*}{ Subsequent surgery in success cases } & $\begin{array}{l}\text { Optical PKP } \\
\text { LT }\end{array}$ & 1 & $\begin{array}{ll}\text { Enucleation } & 1 \\
\text { (painful blind eve) }\end{array}$ & Optical PKP & 2 & \\
\hline & & & $\begin{array}{ll}\text { Exenteration } & 1 \\
\text { (advanced tumour) }\end{array}$ & & & \\
\hline Additional management in failure cases & $\mathrm{LT}+\mathrm{PKP}$ & 1 & AntiHSV Rx 1 & Tectonic graft & 1 & \\
\hline AMT intact $(M)$ & $\begin{array}{l}\text { Antifungal Rx } \\
6.1+/-7.1 \\
(0.25-24)\end{array}$ & 1 & $2.4+/-3.8(0.25-13)$ & $2.5+/-1.2(1-4)$ & & 0.028 (Kruskal-Wallis test) \\
\hline
\end{tabular}

AMT = amniotic membrane transplantation, $\mathrm{ED}=$ epithelial defect, $\mathrm{PKP}=$ penetrating keratoplasty, $\mathrm{LT}=$ limbal transplantation, $\mathrm{HSV}=$ herpes simplex virus, $\mathrm{Rx}$ $=$ treatment.

visual acuity was limited to equal or worse than $6 / 60$ in 23 cases $(82.1 \%)$.

In all patients, conventional medical and surgical treatments to correct the underlying and associating conditions had been tried. Non-preservative artificial tears (18 cases) and punctal occlusion (seven cases) for sicca patients, applications of extended bandage lens (nine cases), pressure patching (six cases), and/or tarsorhaphy (three cases, Fig 1B, D, F) were performed in patients with exposure keratitis and neurotrophic ulcer (details are in Tables 1 and 2). Despite all the efforts, epithelial defects persisted for 6.9 (7.0) weeks (2-24 weeks) in group A and 4.0 (3.3) weeks (1-12 weeks) in group B. In group C, no conventional therapy had been tried because of corneal perforations which mandate to immediate surgery.

Single layer AMT was performed in group A (Fig 1D-F) and a multilayer method was performed in almost all patients with corneal thinning and/or perforation (groups B (Fig 2A) and $\mathrm{C}(\mathrm{Fig} 3 \mathrm{~B}, \mathrm{C})$ ) to promote epithelial healing and restore corneal integrity. Glue had been used to fill the bottom of the perforated ulcer in one case (group C, Fig 3E, F) before placing multiple layers of the amniotic membrane. Some cases required repeated transplantation which will be discussed later.

The total success rate of epithelial healing was $82.1 \%$ ( 23 of 28 eyes), with $80 \%$ (eight of 10 eyes), $84.6 \%$ ( 11 of 13 eyes), and $80 \%$ (four of five eyes), in groups $\mathrm{A}, \mathrm{B}$, and $\mathrm{C}$ respectively. In terms of epithelialisation, the average sizes of corneal epithelial defect in each group before the surgeries were not significantly different $(3.9,3.5$, and $4.3 \mathrm{~mm}$ in group $\mathrm{A}, \mathrm{B}$, and $\mathrm{C}$ respectively, $\mathrm{p}=0.44)$. The total mean epithelialisation time after AMT was 2.1 (SD 1.9) weeks (range $0.5-8$ weeks), with 3.5 (2.6) weeks in group A, 1.5 (1.0) weeks in group B and $1.1(0.5)$ weeks in group C. Among these three groups, epithelial defects in groups B and $\mathrm{C}$ healed more rapidly than those in group $\mathrm{A}(\mathrm{p}$ $=0.003, p=0.017$, and $p=0.009)$ (Table 3).

Among the successful cases, all but three $(89.3 \%)$ healed within 4 weeks. The cases with delayed healing were a case of partial limbal deficiency from Stevens-Johnson syndrome which took 6 weeks to heal, an undetected severe dry eye patient who healed rapidly within a week after punctal occlusion was additionally performed, and a case of severe neurotrophic keratopathy which required 8 weeks to recover.

Regarding the techniques performed, epithelial defects of the patients who underwent multilayer AMT healed significantly faster than those who underwent a single layer method ( $\mathrm{p}$ $=0.014)$. The stromal thickness of the patients with corneal stromal thinning and/or perforation (groups B and C) also significantly increased at the end of the follow up ( $\mathrm{p}=$ 0.022 , Table 3 ). These statistically significant findings might relate to the multilayer method AMT used in groups B and C, which will be discussed later.

Most of the patients already had limited visual acuity due to their corneal abnormalities, thus the slight opacification of the amniotic membrane during the first few weeks did not cause their vision to deteriorate further. Furthermore, the membrane became gradually transparent and the healing area became clearer, similar to the adjacent area. Interestingly, the amniotic membrane in group A persisted longer than that in group B and C (Table 3). However, $66 \%$ of the amniotic membrane dissolved before 3 months.

Ten cases required repeated AMT, four were failed cases and six were successful cases. 
Repeated operations were required in the six successful cases because of new epithelial defects that occurred at the different site after the initial ones were healed (four cases). Those are cases with lid abnormality (Fig 1A, B), post-radiation, and severe neurotrophic keratopathy. One successful case required repeated AMT due to glue removal after the defect was completely healed (Fig 3E-H), the other from graft lost (case 6 in group B, Table 2). Among all the successful cases that underwent repeated AMT, the epithelial healing time in patients who had single transplantation was not different from those underwent multiple transplantations.

Failure was noted in five cases $(17.8 \%)$ in this study, two from undetected active infection, two from severe neurotrophic keratopathy with and without limbal deficiency, and one had intractable corneal perforation. Among the two infected cases, one eye had an occult fungal corneal ulcer at the time of AMT and the other eye developed geographic herpetic ulcer after AMT. Infection in both cases responded well to medical treatment but the defects persisted. Four of these five failed cases underwent repeated AMT without success.

\section{Discussion}

The basic principles for treating PED can be summarised as follows: firstly, correction of mechanical insults such as lid abnormality and exposure. Secondly, surface healing should be promoted by punctal occlusion and artificial tears, ${ }^{15}$ application of growth factors, ${ }^{5}$ and substance P. ${ }^{6}$ Thirdly, inflammation should be suppressed by using topical steroids and antiproteolytic substances. ${ }^{16}{ }^{17}$

Amniotic membranes have been widely used in ophthalmology: as a graft and patch for conjunctival reconstruction, ${ }^{18-21}$ corneal surface reconstruction, ${ }^{11-14}$ 20-24 forniceal reconstruction, ${ }^{18}$ and glaucoma surgery. ${ }^{25}$ The membrane possesses several properties that serve all basic principles for managing PED. It acts as a biological bandage lens to prevent mechanical trauma from lids, prevent exposure, and reduce surface dryness. Amniotic membrane has been reported by several investigators to promote ocular surface healing and can be successfully used in PED. ${ }^{11-13}$ Our study not only supports the idea that AMT can be considered as an effective therapy for treating PED but also demonstrated that this procedure is effective in treating corneal perforation associated with PED. The success rates are some $80 \%$ in total $(80 \%, 84.6 \%$, and $80 \%$ in groups $\mathrm{A}, \mathrm{B}$, and C respectively), regardless of stromal thickness. The epithelialisation times were also shorter after AMT. This finding might be explained by the fact that amniotic membrane provides a thick basement membrane to facilitate epithelial migration and reinforces the adhesion of basal epithelial cells ${ }^{26}{ }^{27}$ causing rapid epithelialisation. It also promotes epithelial differentiation and prevents epithelial apoptosis. ${ }^{28}{ }^{29} \mathrm{~A}$ recent study also shows that preserved AM expresses mRNAs for several growth factors and contains a number of growth factor proteins that might benefit epithelialisation. ${ }^{30}$
In this study, we reported the efficacy of AMT alone (three cases) and combined treatment with glue (one case) in treating small corneal perforations, while $\mathrm{Su}$ and $\mathrm{Lin}^{31}$ previously reported a satisfactory result of combined glue and amniotic membrane in a case of corneal perforation, using a different technique.

Among the three groups in our study, with no different of epithelial defect size before the surgeries $(p=0.44)$, epithelial defects in groups B (PED with stromal thinning) and C (with perforation) healed more rapidly than those in group A ( $p=0.017$ and 0.009). Regarding the techniques performed, patients with multilayer AMT healed faster than those with a single layer. This difference was statistically significant $(p=0.014)$. Since most of the patients in groups B and C underwent multilayer AMT while patients in group A received a single layer technique, we relate these findings to the aforementioned studies which suggest that the amniotic membrane matrix provided the growth factors needed for epithelial healing. It is possible that the more amniotic membrane matrix placed on the ulcer, the faster the wound healing.

Interestingly, corneal stromal thickness in this study (groups B (Fig 2C, F) and C (Fig $3 \mathrm{~A}, \mathrm{D})$ ) can be significantly restored in all cases $(p=0.022)$. This finding is consistent with the study of Kruse $^{12}$ and might be explained by his hypothesis that AM might be able to modify the proliferation and migration of stromal fibroblasts. It should be noted that a cornea with a perforated ulcer can recover to normal or near normal thickness with a significant amount of stromal keratocytes as shown in the pathological section (Fig $3 \mathrm{H}$ ). Furthermore, the rate of amniotic membrane dissolution is faster in groups B and C compared with group A. It is possible that with a stromal ulcer, the inflammatory cytokines and proteolytic enzymes released by stromal keratocytes and inflammatory cells are more abundant thus inducing more rapid membrane dissolution.

In this study, ocular surface inflammation was markedly reduced after AMT, which has been reported previously by several investigators. ${ }^{11-13}$ This may be explained by studies showing that the stromal matrix of the amniotic membrane excludes inflammatory cells, ${ }^{32}$ contains protease inhibitors ${ }^{33}$ and antiinflammatory protein, ${ }^{34}$ suppresses TGF- $\beta$ signalling and proliferation, and myofibroblast differentiation of human corneal and limbal fibroblasts. $^{3536}$

Failure was noted in five cases $(17.8 \%)$ in this study, two from undetected active infection, two from severe neurotrophic keratopathy with and without limbal deficiency, and one had an intractable corneal perforation. These failures point out the limitations of AMT in treating PED. Firstly, continuous tissue destruction can be anticipated with active infection underneath the graft and this will retard healing. Secondly, adequate limbal stem cells and intact sensory innervation are mandatory for repairing and maintaining the ocular surface integrity. ${ }^{13}$ Thirdly, normal keratocytes from the adjacent area might be important in 
restoring stromal integrity after AMT, as demonstrated in one of our failed cases in which a perforation occurred at the centre of the scarred area. A multilayer AMT was performed without success, presumably because of the lack of healthy keratocytes needed for wound healing.

An amniotic membrane graft possesses many advantages over a conjunctival graft. The membrane usually becomes transparent after a few weeks causing no deterioration in visual acuity. Unlike a conjunctival flap or graft, the cornea covered by amniotic membrane will develop less vascularisation and is more acceptable in term of cosmesis. Furthermore, unnecessary trauma at the donor site can be avoided by using an amniotic graft, leaving a healthy conjunctiva for future surgery.

Lee and Tseng ${ }^{11}$ first demonstrated the efficacy of AM in treating PED from various causes while Kruse $^{12}$ successfully introduced the use of multilayer AMT for deep corneal ulcer. In this study, we reported the results of AMT for a variety of corneal ulcer, using single and multilayer technique. We have demonstrated that AMT is an effective treatment for PED and PED with deep ulcer, descemetocele, and perforation. Performing multilayer AMT can successfully manage small corneal perforations and convert emergency tectonic PKP into elective optical PKP which is a more favourable procedure with a better outcome for vision. The use of AMT in treating PED with deep ulcer and perforation might even eliminate the need for PKP in some cases and is therefore beneficial in the countries where cornea shortage is apparent. The technique is simple and produces no additional serious adverse effect to the patients. We therefore encourage the use of AMT in simple and complicated PED. However, there is no established consensus regarding the optimal time to perform AMT in corneal epithelial defects. Should the method be delayed until all conventional treatments fail or be considered earlier? We recognise that our series is relatively small and a larger, randomised, controlled study may be needed to answer all these questions.

The authors wish to thank the Bangkok Biomaterial Center for preparing and providing the amniotic membrane, the Department of Obstetrics and Gynecology, Siriraj Hospital, for helpful cooperation in obtaining the placenta, associate professor Thaivadee Dulayajinda and associate professor Panida Kosrisukvongs for their helpful advice.

1 Macaluso DC, Feldman ST. Pathogenesis of sterile corneal erosions and ulcerations. In: Krashmer JH, Mannis MJ, Holland EJ, eds. Cornea, fundamentals and cornea and external disease. Missouri: Mosby, 1997;204-5.

2 Berman M. The pathogenesis of corneal epithelial defects. Acta Ophthalmol (Copenh) (Suppl) 1989;67:55-64.

3 Spigelman AV, Deutsch TA, Sugar J. Application of homologous fibronectin to persistent human corneal epithelial defects. Cornea 1987;104:494-501.

4 Nishida T, Nakagawa S, Manabe R. Clinical evaluation of fibronectin eyedrops on epithelial disorders after herpetic keratitis. Ophthalmology 1985;92:213-6.

5 Feldman ST. The effect of epidermal growth factor on corneal wound healing: practical considerations for therapeutic use. Refract Corneal Surg 1991;7:232-9.

6 Brown SM, Lamberts DW, Reid TW, et al. Neurotrophic and anhidrotic keratopathy treated with substance P and insulinlike growth factor I. Arch Ophthalmol 1997;115:926-
7 Golubovic S, Parunovic A. Cyanoacrylate glue in the treatment of corneal uclerations. Fortschr Ophthalmol 1990;87: ment of

8 Pfiser RR. Clinical measures to promote corneal epithelial healing. Acta Ophthalmol (Copenh) 1992;70:78-83.

9 Lugo M, Arentsen JJ. Treatment of neurotrophic ulcers with conjunctival flaps. Am f Ophthalmol 1987;103:711-2.

10 Welch C, Baum J. Tarsorrhaphy for corneal disease in patients with rheumatoid arthritis. Ophthalmic Surg 1988; 19:31-2.

11 Lee S, Tseng SCG. Amniotic membrane transplantation for persistent epithelial defects with ulceration. Am $\mathcal{F}$ Ophthalmol 1997;123:303-12.

12 Kruse FE, Rohrschneider K, Volcker HE. Multilayer amniotic membrane transplantation for reconstruction of deep otic membrane transplantation for reconstruction

13 Chen HJ, Pires TF, Tseng SCG. Amniotic membrane transplantation for severe neurotrophic corneal ulcers. $\mathrm{Br} \mathcal{F}$ Ophthalmol 2000;84:826-33.

14 Prabhasawat P, Kosrirukvong P, Booranapong W, et al. Application of preserved human amniotic membrane for corneal surface reconstruction. Cell Tis Bank 2000;1:21322.

15 Tseng SCG. Tsubota K. Important concepts for treating ocular surface and tear disorders. Am $\mathcal{F}$ Ophthalmol 1997;124:825-35.

16 Newsome DA, Gross J. Prevention by medroxyprogesterone of perforation in the alkali-burned rabbit cornea: inhibition of colla 31 .

17 Pfister RR, Haddox JL, Dadson RW, et al. Polymorphonuclear leukocytic inhibition by citrate, other metal chelators, and trifluoperazine. Invest Ophthalmol Vis Sci 1984;25:95570 .

18 Tseng SCG, Prabhasawat P, Lee S. Amniotic membrane transplantation for conjunctival surface reconstruction. Arch Ophthalmol 1997;124:765-74.

19 Shimazaki J, Shinozaki, Tsubota K. Transplantation of amniotic membrane and limbal autograft for patients with recurrent pterygium associated with symblepharon. $\mathrm{Br} \mathcal{f}$ Ophthalmol 1998;82:235-40.

20 Prabhasawat P, Barton K, Burkett G, et al. Comparison of conjunctival autografts, amniotic membrane grafts and primary closure for pterygium excision. Ophthalmology 1997 ; 104:974-85.

21 Dua HS, Azuara-Blanco. Amniotic membrane transplantation. Brf Ophthalmol 1999;83:748-52.

22 Tsubota K, Satake Y, Ohyama M, et al. Surgical reconstruction of the ocular surface in advanced ocular cicatricial tion of the ocular surface in advanced ocular cicatricial
pemphigoid and Stevens-Johnson syndrome. Am $\mathcal{F} O p h-$ thalmol 1996;122:38-52.

23 Shimazaki J, Hao-Yang Y, Tsubota K. Amniotic membrane transplantation for ocular surface reconstruction in patients with chemical and thermal burns. Ophthalmology 1997;104:2068-76.

24 Tseng SCG, Prabhasawat P, Barton K, et al. Amniotic membrane transplantation with or without limbal allografts for corneal surface reconstruction in patients with limbal stem cell deficiency. Arch Ophthalmol 1998;116:431-41.

25 Fujishima H, Shimazaki J, Shinozaki N, et al. Trabeculecomy with the use of amniotic membrane for uncontrollable glaucoma. Ophthalmic Surg Lasers 1998;29:428-31.

26 Khodadoust AA, Silverstein AM, Kenyon KR, et al. Adhesion of regenerating corneal epithelium: the role of Adhesion of regenerating corneal epithelium: the role of
basement membrane. Am f Ophthalmol 1968;57:311-7.

27 Sonnenberg A, Calafat J, Janssen $\mathrm{H}$, et al. Integrin a6/b4 complex is located in hemidesmosomes, suggesting a major role in epidermal cell-basement membrane adhesion. 7 Cell Biol 1991;113:907-17.

28 Boudreau N, Sympson CJ, Werb Z, et al. Suppression of ICE and apoptosis in mammary epithelial cells by extracellular matrix. Science 1995;267:891-3.

29 Boudreau N, Werb Z, Bissell MJ. Suppression of apoptosis by basement membrane requires three-dimensional tissue organization and withdrawal from the cell cycle. Proc Natl Acad Sci USA 1996;93:3500-13.

30 Koizumi N, Inatomi T, Sotozono C, et al. Growth factor mRNA and protein in preserved human amniotic membrane. Curr Eye Res 2000;20:173-7.

$31 \mathrm{Su}$ CY, Lin CP. Combined use of an amniotic membrane and tissue adhesive in treating corneal perforation: a case report. Ophthalmic Surg Lasers 2000;31:151-4.

32 Park WC, Tseng SCG. Temperature cooling reduces keratocyte death in excimer laser ablated corneal and skin wounds. Invest Ophthalmol Vis Sci 1998;39:S449.

$33 \mathrm{Na}$ BK, Hwang JH, Shin EJ, et al. Analysis of human amniotic membrane components as proteinas inhibitors for development of therapeutic agent of recalcitrant keratitis. Invest Ophthalmol Vis Sci 1998;39:S90.

34 Hao Y, Hui-Kang D, Hwang D, et al. Identification of antiangiogenic and antiinflammatory proteins in human amniotic membrane. Cornea 2000;19:348-52.

35 Tseng SCG, Li D, Ma X. Suppression of transforming growth factor beta isoforms, TGF- $\beta$ receptor type II, and myofibroblast differentiation in cultured human corneal and limbal fibroblasts by amniotic membrane matrix. $f$ Cell Physiol 1999;179:325-35.

36 Lee S, Li D, Tan DTH, et al. Suppression of TGF- $\beta$ signaling in both normal conjunctival fibroblasts and pterygiual body fibroblasts by amniotic membrane. Curr Eye Res 2000;20:325-34. 